\title{
MODELOS DE ANÁLISE DAS POLÍTICAS PÚBLICAS
}

\author{
Luísa Araújo \\ FPP - Fórum das Políticas Públicas, Lisboa, Portugal
}

Maria de Lurdes Rodrigues

Instituto Universitário de Lisboa (ISCTE-IUL), Centro de Investigação e Estudos de Sociologia (CIES-IUL), Lisboa, Portugal

\begin{abstract}
Resumo É objetivo deste artigo apresentar e discutir quatro modelos teóricos que contribuem para a compreensão das políticas públicas: (1) o modelo sequencial ou do ciclo político, (2) o modelo dos fluxos múltiplos, (3) o modelo do equilíbrio interrompido e (4) o quadro teórico das coligações de causa ou de interesse. Estes modelos são considerados por vários autores como sendo os quadros teóricos mais promissores no campo analítico das políticas públicas. São modelos logicamente coerentes, empiricamente verificáveis, claros e abrangentes e replicáveis em diferentes áreas de política, situações e contextos.
\end{abstract}

Palavras-chave: políticas públicas, processo político, ação pública, modelos de análise das políticas públicas.

\begin{abstract}
In this paper, the objective is to present and discuss four theoretical models that contribute to the understanding of public policies: (1) the Policy Cycle Model, (2) the Multiple Streams Framework, (3) the Punctuated Equilibrium Theory, and (4) the Advocacy Coalition Framework. These models are considered by many authors as the most adequate analytical frameworks in the scientific field of Public Policy. The models are logically consistent and empirically verifiable, clear and comprehensive, replicable in different policy areas, situations and contexts.
\end{abstract}

Keywords: public policies, policy process, public action, public policies theoretical framework.

Résumé L'objectif de cet article est de présenter et de débattre quatre modèles théoriques qui contribuent à la compréhension des politiques publiques: (1) le Modèle séquentiel ou du Cycle politique, (2) le Modèle des flux multiples, (3) le Modèle de l'équilibre ponctué et (4) le Cadre théorique des coalitions de cause ou d'intérêt. Ces modèles sont considérés par plusieurs auteurs comme les cadres théoriques les plus promettants dans le domaine analytique des politiques publiques. Ces modèles sont logiquement cohérents et vérifiables empiriquement, ils sont claires et complets et reproductibles dans différents domaines de la politique, dans des situations et des contextes différents.

Mots-clés: politique publique, processus politique, intervention publique, modèles d'analyse des politiques publiques.

Resumen El propósito de este artículo es presentar y discutir cuatro modelos teóricos que contribuyen a la comprensión de las políticas públicas: (1) modelo secuencial o del ciclo político, (2) modelo de flujos múltiples, (3) modelo del equilibrio puntuado y (4) cuadro teórico de las coaliciones de causa o interés. Estos modelos son considerados por muchos autores como los cuadros teóricos más prometedores en el campo de análisis de las políticas públicas. Los modelos son lógicamente consistentes y empíricamente comprobables, claros y comprensivos y replicables en diferentes ámbitos políticos, situaciones y contextos.

Palabras-clave: política pública, proceso político, la acción pública, los modelos de análisis de políticas públicas.

A análise das políticas públicas tem como objeto de estudo as decisões políticas e os programas de ação dos governos, interrogando-se sobre a génese dos problemas que tais decisões procuram resolver, sobre as soluções formuladas e as condições da sua implementação. As políticas públicas, enquanto objeto de estudo, configuram, em 
primeiro lugar, processos complexos e multidimensionais que se desenvolvem em múltiplos níveis de ação e de decisão - local, regional, nacional e transnacional. Em segundo lugar, envolvem diferentes atores - governantes, legisladores, eleitores, administração pública, grupos de interesse, públicos-alvo e organismos transnacionais -, que agem em quadros institucionais e em contextos geográficos e políticos específicos, visando a resolução de problemas públicos, mas também a distribuição de poder e de recursos.

Como veremos, a análise das políticas públicas, ao tomar como objeto de estudo a ação pública afirma-se, em ciência política, como a disciplina que permite abrir a "caixa negra" do sistema político. Tradicionalmente a ciência política tem como objeto de estudo as questões do poder, como este é conquistado, mantido, distribuído e partilhado, os mecanismos de reprodução e de aquisição de recursos de poder, bem como os processos de participação, de competição e de alianças. Neste quadro, a análise da atividade dos governos e das instituições do sistema político administrativo não é considerada relevante.

A análise das políticas públicas parte de contributos teóricos gerais da ciência política, mas também da economia, da psicologia, da sociologia, da história e dos estudos das organizações. Porém, define um campo de estudos específico pluridisciplinar e abre espaço ao desenvolvimento de teorias de médio alcance, modelos, mapas, metáforas e conceitos próprios, que permitem explicar e pensar as políticas públicas, permitem compreender os modos e as regras gerais de funcionamento da ação pública e analisar as suas continuidades e ruturas, bem como os processos e as determinantes do seu desenvolvimento, e identificar a multiplicidade de fatores e forças que formam os processos reais das políticas públicas. O seu objetivo não é explicar o funcionamento do sistema político, mas a lógica da ação pública, as continuidades e ruturas nas políticas públicas, as regras do seu funcionamento, a afetação de recursos e o papel e os modos de interação de atores e instituições nos processos políticos.

A análise das políticas públicas, enquanto ciência social, beneficiou no seu desenvolvimento de quadros teóricos e de conceitos de outras disciplinas, sendo vasto o seu património teórico sistematizado em obras de referência, como por exemplo Parsons (1995), Hill (2009), Knoepfel et al. (2011), Sabatier (2007) e Fischer (2003).

Propomo-nos neste texto rever os contributos teóricos dos fundadores da disciplina e apresentar e discutir quatro modelos teóricos de análise das politicas públicas.

Os quatro modelos apresentados e discutidos neste trabalho são considerados por vários autores como sendo os quadros analíticos mais promissores, ${ }^{1}$ dadas as suas características. São eles: (1) o modelo sequencial ou do ciclo político, (2) o modelo dos fluxos múltiplos, (3) o modelo do equilíbrio interrompido e (4) o quadro teórico das coligações de causa ou de interesse. Trata-se de modelos teóricos logicamente coerentes, baseados em proposições empiricamente verificáveis, claros

1 Ver, por exemplo, Sabatier (2007), Muller (2010), Capella (2006), Birkland (2011). 
e abrangentes, replicáveis em áreas de política, situações e contextos distintos. Apresentam-se como modelos de síntese, uma vez que procuram promover a integração de conceitos e propostas analíticas de outros autores, explorando a complementaridade das diferentes abordagens.

\section{Emergência da análise das políticas públicas}

A análise de políticas públicas como campo disciplinar de estudo surge e desenvolve-se nos EUA, ${ }^{2}$ no pós-guerra, em condições políticas, económicas e sociais particulares. ${ }^{3}$ Por um lado, assistia-se ao alargamento das áreas de intervenção do estado na resolução de problemas, exigindo crescentemente informação sobre os mais variados setores, da educação à saúde, aos transportes e planeamento urbano, à defesa e segurança (Parsons, 1995: 20). Por outro lado, nas universidades americanas, entre académicos e investigadores de ciência política difunde-se uma orientação favorável ao desenvolvimento de conhecimento e informação necessários a uma "boa" governação, tendo em vista o sucesso e eficiência das políticas públicas na melhoria das condições de vida dos cidadãos. Defende-se então a aplicação de métodos científicos às decisões do governo em todas as áreas de intervenção. Cultiva-se também uma preocupação com a promoção das práticas da democracia (DeLeon, 2006: 40-41). O trabalho de Lasswell, um dos fundadores da disciplina, é a vários títulos um exemplo desta orientação. ${ }^{4}$

Os cientistas sociais norte-americanos Harold Lasswell, Herbert Simon, Charles Lindblom e David Easton, são considerados os "fundadores" do estudo das políticas públicas, como área científica autónoma, pelos trabalhos seminais desenvolvidos por volta dos anos 50 (Fisher, 2003; DeLeon, 2006; Parsons, 1995; Hill, 2009; Sabatier, 2007).

Harold Lasswell (1948) introduz pela primeira vez a expressão policy analysis (análise de políticas públicas), afirmando a análise do processo político como objeto de estudo alternativo aos objetos tradicionais da ciência política, isto é, alternativo ao estudo das constituições, legislaturas, grupos de interesse, elites e questões clássicas do poder.

A sua obra contribui de forma decisiva, em primeiro lugar, para a estruturação do campo de análise das políticas públicas como uma ciência social aplicada e, em segundo lugar, para lançar as bases do que virá a ser o modelo de análise sequencial ou das etapas do processo político.

2 Na Europa, a disciplina desenvolve-se apenas a partir de meados dos anos 80, ancorada em perspetivas e focos de análise muito diferentes (Muller, 2005).

3 DeLeon e Vogenbeck (2007) argumentam que a crescente complexidade da ação pública, decorrente dos múltiplos e diversificados contextos políticos, económicos e sociais que emergiram no período pós-guerra, estiveram muito associados à consolidação e desenvolvimento do campo da análise das políticas públicas, cujas abordagens, eminentemente multidisciplinares, transcendem os contributos individuais de outras áreas de estudo, designadamente da ciência política, da sociologia, da administração pública, da comunicação, da psicologia e da jurisprudência. Sobre o legado de Lasswell, ver o texto de D. Torgerson (2007). 
Ao longo da sua carreira este autor desenvolve intenso trabalho de consolidação da ideia de uma ciência política e de um papel para os analistas políticos. Com base no pressuposto analítico de que aquilo que o estado faz ou deixa de fazer pode ser formulado cientificamente por investigadores independentes, encarando a disciplina como subsidiária dos contributos da ciência política, da sociologia, da antropologia, da psicologia, da estatística, da matemática e mesmo das ciências exatas, Lasswell ambicionava desenvolver uma ciência da formulação e concretização das políticas, marcadamente normativa, que, ancorada em abordagens multidisciplinares e em metodologias qualitativas e quantitativas, habilitasse os decisores políticos com a informação necessária à sua ação e contribuísse para aumentar a racionalidade do processo de tomada de decisão ou, segundo o próprio, uma ciência baseada no conhecimento "no e do processo político" (in and of the policy process).

O cientista político contemporâneo percebe-se a si mesmo como um integrador de conhecimento e ação [...]. Um mediador entre os especialistas de áreas específicas de conhecimento, os decisores políticos e os cidadãos. (Lasswell, 1970: 3-14)

Lasswell foi um dos primeiros autores, em 1956, a tentar estabelecer/formular o conjunto de etapas de desenvolvimento do processo político, propondo uma classificação em sete etapas: "informação" (recolha de dados); "iniciativa" (aprovação de medidas de política); "prescrição" (formulação de medidas, normas e regras); "invocação" (justificação e especificação dos benefícios e das sanções); "aplicação" (concretização das medidas); "avaliação" (sucesso ou insucesso das decisões), e "cessação" (regras e instituições criadas no âmbito da política aprovada).

Como veremos adiante, esta ideia de etapas ou fases de desenvolvimento do processo político será objeto de debate e reflexão no campo das políticas públicas, ao longo dos 50 anos seguintes. Muitos autores contribuirão para o seu aprofundamento, vindo a proposta de Lasswell a transformar-se no ponto de partida para a construção de quase todos os novos modelos e quadros teóricos da análise de políticas públicas.

Herbert Simon desenvolve um trabalho de natureza multidisciplinar sobre os processos de decisão nas organizações e dá um contributo decisivo para a evolução do campo das políticas públicas, no final da década de 1950. A partir dos seus estudos centrados na análise dos processos de decisão nas organizações, desenvolve e amplia o conceito de "racionalidade limitada dos decisores políticos" (bounded rationality). Argumenta que a capacidade de lidar com os problemas de uma forma racional é sempre limitada por fatores exógenos e endógenos, como a natureza necessariamente fragmentada e incompleta do conhecimento e da informação, a ocorrência de mudanças imprevisíveis de contexto, o limite de tempo disponível para a tomada de decisão, a capacidade limitada da memória humana ou mesmo os valores e interesses próprios.

Considera contudo que a verificação de algumas condições permite melhorar a racionalidade da decisão, designadamente os processos de especialização de indivíduos e organizações, que permitem criar rotinas e standards para decisões que se repetem (Simon, 1983). No mesmo sentido, também os mecanismos de mercado ou os mecanismos de contraditório e os instrumentos técnicos restringem e organizam a 
informação necessária à decisão, melhorando as condições de racionalidade, tal como a informação pública, o conhecimento sobre as instituições políticas e o conhecimento em geral.

Charles Lindblom ${ }^{5}$ dá um contributo decisivo para o desenvolvimento da análise das políticas públicas como processo político. Sendo crítico da ideia, desenvolvida por Simon, de racionalidade no processo de decisão, focou a sua atenção na identificação da margem de manobra dos decisores políticos, que considerava ser muito limitada. Construiu, uma abordagem analítica alternativa que classificou como "método das comparações sucessivas" (method of sucessive limited comparisons) ou "incrementalista".

No seu modelo analítico, Lindblom (1959: 84-86) defende que o processo de decisão política tem as seguintes características: (i) é construído passo a passo, através de mudanças incrementais, com base em políticas preexistentes; (ii) envolve ajustamentos mútuos e negociação, e (iii) não é uma solução final para os problemas, é apenas um passo que, quando é bem-sucedido, pode ser seguido de outros.

As políticas não são construídas de uma só vez; são construídas e reconstruídas interminavelmente. A construção de políticas é um processo de aproximações sucessivas aos objetivos pretendidos, no qual os próprios objetivos vão sendo reconsiderados e alterados. (Lindblom, 1959: 86)

Abrange na sua análise variáveis ignoradas pelas abordagens racionalistas, como as relações de poder, os processos eleitorais e o papel das burocracias, dos partidos e dos grupos de interesse. Por outro lado, defende também a necessidade de uma visão mais integrada das diferentes fases e dimensões do processo político.

Lindblom é crítico em relação ao modelo sequencial de análise do processo político proposto por Lasswell e à ideia da identificação de etapas, argumentando que "fases sequenciais e ordenadas deliberadamente não são uma forma rigorosa de retratar o modo como funciona o processo político (Lindblom e Woodhouse, 1993), e considerando que tanto o modelo sequencial como o modelo da escolha racional serviram mais para obscurecer do que para iluminar o processo político, demasiado complexo para ser apreendido por modelos excessivamente simplificadores.

David Easton (1957) desenvolve a aplicação da abordagem sistémica à análise das políticas públicas, tendo tido também uma influência decisiva na evolução da disciplina. Conceptualiza a relação entre o processo político, as políticas públicas e o respetivo contexto social, económico e político. As políticas públicas são, no seu modelo, um output do sistema político, revelador da emergência, da natureza e da atividade do estado. Neste sentido, Easton entende o processo político como um sistema em que cada componente não pode ser analisado isoladamente: a ação de cada um dos intervenientes no processo político só

5 O seu texto publicado em 1959, “The science of 'muddling through'”, é um dos textos fundadores mais importantes. 
pode ser adequadamente percebida quando inserida no todo. No sistema políti$\mathrm{Co}$, atores e instituições políticas interagem, enquadrados por um conjunto de normas, símbolos e valores, cujo comportamento é condicionado quer por mecanismos de suporte da envolvente social (inputs), quer por pressões internas ao sistema (withinputs) que, para além de influenciarem o comportamento do sistema, também o alimentam e mantêm ativo, através da apresentação de exigências, necessidades e problemas, por intermédio dos input channels (partidos, média, grupos de interesses).

Segundo Easton, inputs e withinputs não se transformam automaticamente em problemas políticos: a notoriedade das exigências, os jogos de poder no exterior e no interior do sistema, a existência de competências políticas e técnicas para lidar com as exigências são algumas das condições para a emergência de um problema político no interior do sistema. Uma vez identificados, os problemas são processados, nomeadamente através de mecanismos de regulação, distribuição e redistribuição, dando origem a outputs - as decisões políticas, que se constituem como resposta às necessidades e exigências apresentadas. Num processo de feedback, os outputs podem dar origem a novos inputs, o que confere ao modelo proposto por Easton uma dinâmica cíclica e inacabada.

As diferenças nas políticas públicas são explicadas pelas diferenças nas instituições do estado e pelas diferenças do funcionamento do sistema político. O processo político de tomada de decisão e de iniciativa é entendido, neste quadro, como uma "caixa negra".

Apesar de distintas, as abordagens propostas por Lasswell, Simon, Lindblom e Easton têm em comum três características que contribuíram para conferir um caráter distintivo ao campo científico das políticas públicas: ${ }^{6}$

- são explicitamente orientadas para os problemas públicos e para as suas soluções (problem oriented); os problemas ocorrem em contextos específicos, que devem ser considerados quer na sua análise, quer na escolha das soluções;

- são distintivamente multidisciplinares, nas suas abordagens teóricas e práticas, o que é justificado pelo facto de a maioria dos problemas políticos integrarem múltiplos componentes, ligados a várias disciplinas, as quais são relevantes para uma completa análise e compreensão dos fenómenos políticos;

- são orientadas por valores: o ethos democrático e a dignidade humana ocupam um lugar central na análise das políticas públicas;

- afirmam a possibilidade de, nas sociedades democráticas, a ação - ou inação - dos decisores políticos ser analisada e formulada cientificamente, por cientistas independentes.

Os quadros teóricos que dominaram o campo da análise das políticas públicas, a partir dos anos 60, derivam da combinação das abordagem lançadas por estes autores, configurando-se desde muito cedo duas grandes correntes de pensamento e

$6 \quad$ Ver Peter DeLeon (2006: 53-54). 
de análise, dois caminhos analíticos paralelos, mas também complementares, com muitos pontos de contacto e de cruzamento.

Por um lado, modelos teóricos resultantes de uma combinação entre a abordagem sequencial (Lasswell) e a abordagem sistémica (Easton), centrados na preocupação de responder à questão de como surgem e como funcionam as políticas públicas, entendidas como um processo com especificidades próprias não explicadas apenas pelo funcionamento do sistema político. Orientam o debate teórico entre os vários autores questões sobre o "funcionamento das políticas públicas": Como surgem e se desenvolvem as políticas públicas? Como emergem os problemas e se processa o seu agendamento político? Que soluções são formuladas, como e porquê? Que decisões são tomadas, por quem, como e porquê? Como são concretizadas as políticas públicas e por quem? Quais as condicionantes dos processos de concretização? Quais os resultados e impactos das decisões? Nesta corrente distinguem-se: (1) as abordagens centradas sobretudo na emergência dos problemas, no processo de agendamento e de formulação das políticas públicas, e (2) as abordagens centradas sobretudo nos processos de concretização das políticas públicas.

Por outro lado, modelos teóricos derivados da combinação entre a abordagem sequencial (Lasswell), a abordagem da escolha racional (Simon) e a abordagem incrementalista (Lindblom), centrados no "processo de decisão". ${ }^{7}$ Orientam o debate teórico entre os vários autores as seguintes questões: Quem decide, como decide e porquê? Quem participa na decisão? Quais as condicionantes da decisão? Qual a relação entre a "política e as políticas públicas"? Neste último caso trata-se sobretudo de propostas de análise das políticas públicas baseadas nas teorias do estado, sendo aquelas instrumentais para a verificação de hipóteses dessas teorias.

Em anexo, apresenta-se uma sistematização dos quadros teóricos e dos modelos de análise desenvolvidos pelos diferentes autores, considerando o seu principal foco de atenção. ${ }^{8}$

Pode dizer-se que os diferentes modelos de análise têm em comum a preocupação de abrir e compreender a "caixa negra" do sistema político, isto é, compreender a ação pública dos governos, as condições do seu desenvolvimento, as suas causas e condicionantes, os fatores de sucesso e de insucesso, as consequências e os efeitos dessa ação, o papel dos atores, o papel das ideias e dos fatores cognitivos, a influência dos fatores socioeconómicos e dos organismos internacionais, procurando padrões e explicações causais através da análise das instituições, dos atores,

7 Ver revisão de literatura sobre abordagens teóricas baseadas nas teorias da escolha racional em M. Levi (2009).

8 Trata-se de uma proposta de sistematização dos diferentes quadros teóricos e modelos de análise das políticas públicas, visando orientar a leitura do extenso património da disciplina. Utilizou-se como critério principal o tema específico ou a questão de análise a que procuram responder os autores das diferentes abordagens. Esta proposta de classificação é apenas um guia, devendo ser utilizada tendo em atenção o facto de, em muitos casos, os autores promoverem estudos que envolvem mais do que uma questão, isto é, mais do que um foco de observação e reflexão, ou procurarem construir abordagens integradas. 
das ideias e de outras variáveis de natureza cognitiva, bem como da análise dos fatores socioeconómicos.

Nos diferentes modelos varia o foco e o objeto de análise em temas específicos, variam as questões de análise (podendo ser mais restritas ou abrangentes), as perspetivas disciplinares e as metodologias de análise, mas estes não são na maioria dos casos mutuamente exclusivos, antes complementares. Como afirma Peter John (1998: 14): "As abordagens não são rivais; podem complementar-se e tornar-se parte da explicação".

Neste artigo apresentaremos quatro dos modelos teóricos de análise de políticas públicas que contribuem para a compreensão da génese dos problemas e do processo das políticas públicas: (1) o modelo sequencial ou do ciclo político, (2) o modelo dos fluxos múltiplos, (3) o modelo do equilíbrio interrompido e (4) o quadro analítico das coligações de causa ou de interesse.

\section{Quatro modelos teóricos para compreender as políticas públicas}

\section{Modelo sequencial ou do ciclo político (Policy Cicle)}

No modelo sequencial, as políticas públicas são tomadas como resultado de um processo que se desenvolve por "etapas, num ciclo político que se repete". Importante não é explicar como funciona o sistema político, mas compreender os modos da ação pública, demonstrar as continuidades e as ruturas, bem como as regras gerais de funcionamento que são específicas das políticas públicas.

Lasswell é um dos primeiros autores a propor, no fim da década de 1950, uma análise estruturada do processo político, sugerindo a sua decomposição em fases sucessivas, relacionadas entre si de uma forma lógica e sequencial. Parte da abordagem sistémica das políticas públicas de Easton para a construção do modelo Policy Cycle ou modelo das etapas, propondo que as políticas públicas sejam analisadas como o resultado de um ciclo político que se desenvolve por etapas, procurando desta forma "abrir a caixa negra" do sistema político. A desagregação em etapas, que podem ser investigadas isoladamente ou em relação com as etapas subsequentes, por redução da complexidade, facilita a compreensão desse mesmo processo (Lasswell, 1956). As designações que Lasswell propõe para cada uma das fases constituem um contributo decisivo para a criação de um mapa conceptual orientador da análise das políticas públicas: informação, promoção, prescrição, invocação, aplicação, conclusão e avaliação.

O modelo sequencial ou do ciclo político permite explorar e investigar o processo das políticas públicas por redução da sua complexidade. A desagregação em etapas ou categorias de análise torna todo o processo das políticas públicas mais facilmente apreensível. Desta forma, a ação pública, orientada para a resolução dos problemas, é analisada como um processo sequencial e inacabado que se repete e reconstrói, em resultado de mudanças induzidas por efeito de feedback das próprias políticas públicas, ou por alterações do contexto ou da relação entre os atores e instituições envolvidos. 
A partir da formulação de Lasswell, nos anos 70, Charles O. Jones (1984) desenvolve uma classificação com dez etapas, mais próxima da que veio a ser consolidada e difundida: perceção do problema; agregação; organização; representação do público-alvo; agendamento; formulação; orçamentação; concretização; avaliação; ajustamento ou conclusão. Muitos outros autores (Jenkins, 1978; Anderson, 2003; Hogwood e Gunn, 1984) apresentaram propostas alternativas de classificação, ${ }^{9}$ contribuindo para a consolidação de um modelo heurístico de decomposição do processo político em sequências ou etapas, para fins analíticos.

Os diferentes autores identificam várias etapas, fases ou sequências no ciclo político, designando-as de formas diferentes, porém quatro etapas são comuns a todas as propostas e podem ser sintetizadas da seguinte forma:

- definição do problema e agendamento, relativos ao contexto e ao processo de emergência das políticas públicas - perceção de um problema como problema político -, ao debate público sobre as suas causas e à entrada do problema na agenda política;

- formulação das medidas de política e legitimação da decisão, relativas ao processo de decisão e de elaboração de argumentos explicativos da ação política, de desenho de objetivos e de estratégias de solução do problema, de escolha de alternativas, bem como de mobilização das bases de apoio político;

- implementação, relativa aos processos de aprovisionamento de recursos institucionais, organizacionais, burocráticos e financeiros para a concretização das medidas de política;

- avaliação e mudança, relativas aos processos de acompanhamento e avaliação dos programas de ação e das políticas públicas, com o objetivo de aferir os seus efeitos e impactos, a distância em relação aos objetivos e metas estabelecidos, a eficiência e eficácia da intervenção pública, os processos de modificação dos objetivos e dos meios políticos decorrentes de novas informações, de alterações no contexto de espaço e de tempo, a partir dos quais (por efeito de feedback) se inicia um novo ciclo político em que as etapas se repetem.

O modelo sequencial ou do ciclo político tem sido alvo de críticas, nomeadamente por se basear numa metodologia de análise muito restrita, e por criar uma visão artificial do processo político.

A título de exemplo, Parsons (1995: 79-80) argumenta que o modelo do ciclo político não é um modelo causal, que não pode ser empiricamente testado e que privilegia uma análise top-down do processo político, ignorando os diferentes níveis de decisão e a diversidade de atores intervenientes no processo, ignorando assim os múltiplos níveis de decisão e de ciclos.

John Kingdon (2011: 205) critica o modelo das etapas, por entender que o processo político não decorre ordenadamente, em fases, referindo, designadamente,

9 May e Wildavksy (1978) compilaram contributos de vários autores para o desenvolvimento do modelo do ciclo político. 
que o agendamento não ocorre em primeiro lugar, gerando posteriormente alternativas. Pelo contrário, para este autor as alternativas são defendidas e ponderadas durante muito tempo, antes que uma oportunidade as coloque na agenda.

Também Paul Sabatier (2007: 7) critica a abordagem, por não identificar o conjunto de causalidades que norteia o processo político, por ser imprecisa, normativa, simplificadora e marcadamente top-down, focando a sua atenção num único ciclo e descurando as dinâmicas decorrentes das interações entre múltiplos ciclos, que envolvem diferentes propostas de solução, diferentes atores e múltiplos níveis de decisão.

Apesar das críticas, o modelo das etapas tem sido o ponto de partida para a maioria das abordagens, metáforas e enquadramentos teóricos da análise das políticas públicas, porque permite relacionar de forma coerente todos os aspetos das mesmas (Muller, 2010: 25). Na realidade, trata-se de um modelo heurístico que permite explorar, para fins exclusivamente analíticos, as políticas públicas. ${ }^{10} \mathrm{Como}$ todos os modelos heurísticos, terá que ser usado com cautela, com a noção clara de que é apenas uma representação simplificada da realidade, cuja função é providenciar um enquadramento analítico facilitador da compreensão do processo político. Pode considerar-se, inclusivamente, que a difusão do seu uso é provavelmente resultado da sua capacidade de proporcionar uma estrutura racional, dentro da qual se pode considerar, para efeitos de análise, a multiplicidade da realidade e em que podem ser aplicados diferentes quadros explicativos. Como refere Pierre Muller,

a representação sequencial das políticas não deve ser utilizada de forma mecânica. É indispensável entender as políticas como um fluxo contínuo de decisões e procedimentos, para os quais é necessário encontrar um sentido. Haverá vantagem em conceber uma política pública não como uma série de sequências sucessivas, mas como um conjunto de sequências paralelas que interagem e se modificam continuamente. (Muller, 2010: 27)

\section{Metáfora dos fluxos múltiplos (Multiple Streams Framework)}

Na sua obra de referência, Agendas, Alternatives and Public Policies, John Kingdon (2011) desenvolve "o modelo analítico Multiple Streams", 11 procurando explicar como é que os problemas se transformam em problemas políticos, isto é, como captam a atenção do público e dos políticos e entram na agenda da ação pública. Nem todas as questões se transformam em problemas políticos suscitando a intervenção do governo, apenas algumas. Como e porquê? O modelo de análise proposto procura responder às seguintes questões: (i) Porque é que os decisores políticos prestam atenção a um determinado assunto em detrimento de outros? (ii) Como e porquê se alteram as agendas políticas ao longo do tempo? (iii) Como é que os decisores

10 Sobre o valor heurístico do modelo das etapas, ver também os argumentos de Michael Hill (2009: 147-148) e Rodrigues (2014).

11 O autor baseia o seu modelo num trabalho de investigação empírica, desenvolvido nos anos 70, nos setores da saúde e dos transportes, que implicou a realização de centenas de entrevistas junto de funcionários públicos e ativistas políticos, bem como a realização de estudos de caso. 
políticos selecionam soluções para os problemas, de entre um vasto conjunto de alternativas?

O autor concebe um modelo baseado numa metáfora e em três conceitos centrais: as comunidades políticas, constituídas por investigadores, deputados, funcionários públicos, analistas, grupos de interesse, cujos membros partilham a preocupação com determinado tema ou problemas e que promovem a construção e a difusão de ideias em diferentes fóruns; os empreendedores políticos, um tipo particular de atores na mediação e negociação dos processos de agendamento; a janela de oportunidade política, que se abre quando convergem três fluxos de variáveis: a perceção pública dos problemas (fluxo dos problemas), o conhecimento de soluções políticas e técnicas adequadas aos valores dominantes (fluxo das políticas) e as condições de governação (fluxo da política). Os três fluxos (streams) fluem autonomamente no sistema político, com regras e dinâmicas próprias:

- Fluxo dos problemas: Kingdon aborda os problemas como construções sociais estabelecidas por intervenção de atores - comunidades políticas - nos processos das políticas públicas. Estabelece uma distinção entre questões e problemas políticos, considerando que uma questão - definida como uma situação socialmente percebida - só se transforma num problema quando os decisores políticos consideram que deve ser encontrada uma solução para a questão. Atendendo ao grande número de questões e à impossibilidade material dos decisores de lidar com todas elas, a emergência de um problema é condicionada por mecanismos que contribuem para que a atenção dos decisores se centre em determinadas questões em detrimento de outras. Esses mecanismos são indicadores, eventos, crises, símbolos e feedback da ação política. ${ }^{12}$ A construção de argumentos, baseados em dados e informações, a elaboração de narrativas sobre as causas dos problemas e as respetivas soluções, a difusão desses argumentos e informações fazem parte da atividade das comunidades políticas, que são mais ou menos fragmentadas, integrando uma diversidade de pontos de vista e de ideias muitas vezes antagónicas. Também os organismos internacionais desempenham neste processo um papel importante. A definição dos problemas constitui uma etapa fundamental, podendo inclusivamente determinar o sucesso da ação política. No entanto, não é suficiente, só por si, para iniciar um processo de agendamento.

- Fluxo das políticas, composto pelo conjunto de alternativas e soluções disponíveis, geradas no interior das comunidades politicas. Para explicar a forma como são geradas as alternativas no interior das comunidades políticas, Kingdon utiliza a metáfora da "sopa primordial" (primeval soup). ${ }^{13}$ Um grande conjunto de ideias fluem no interior das comunidades (policy primeval soup), algumas tornam-se

12 Birkland (2011) analisa também o impacto dos eventos focalizadores no processo das políticas públicas.

13 A teoria da sopa primordial, utilizada em biologia para explicar o aparecimento de vida na Terra, defende que um conjunto de moléculas que "flutuava numa sopa" de matéria orgânica, quando exposto a determinados fatores exógenos, deu origem à vida. 
proeminentes enquanto outras desaparecem, em resultado de um processo evolutivo de amadurecimento, em que as ideias se confrontam e se combinam das mais variadas formas: eventuais consensos decorrem de processos de persuasão e de difusão de ideias. As alternativas de solução não decorrem necessariamente da prévia identificação e perceção política dos problemas; o autor afirma inclusivamente que muitas vezes as soluções são construídas e só posteriormente são identificados os problemas para os quais possam ser aplicadas essas soluções. Não obstante a importância crucial que Kingdon confere às ideias e à existência de alternativas tecnicamente viáveis, considera que estas não representam, só por si, um fator gerador do agendamento.

- Fluxo da política, respeita à dimensão política que segue um curso independente dos problemas e das soluções políticas. O autor inclui neste fluxo três feixes de variáveis decisivas para alterar ou influenciar o processo de agendamento:

- O sentimento nacional (national mood), que caracteriza como "situações em que um grande número de pessoas num país partilha ideias comuns [...] o sentimento nacional sofre mudanças no tempo, de uma forma percetível, e estas mudanças têm importantes impactos nas agendas e nos resultados políticos" (Kingdon, 2011: 146).

- As forças políticas organizadas, onde os atores centrais são os partidos políticos e os grupos de interesse. A perceção que os decisores têm da forma como estes atores se inter-relacionam é de importância crucial para o desenvolvimento das políticas. Se os governos consideram que "a maioria dos grupos de interesse e outras forças organizadas apontam para uma mesma direção, [...] este contexto proporciona-lhes um forte ímpeto para se orientarem nessa direção" (Kingdon, 2011: 150).

- As mudanças governamentais (governmental turnover), nomeadamente mudanças de ciclo político, remodelações e reconfigurações de governos e parlamentos, mudanças nas hierarquias da administração, sendo a mudança de governo o fator mais propício à ocorrência de alterações na agenda política. Ao contrário do que acontece no fluxo das políticas, em que o consenso é produto de processos de persuasão e difusão, no fluxo da política os consensos são construídos através de intensos processos negociais e pelo estabelecimento de coligações.

Como foi já referido, o autor defende que cada um dos fluxos descritos percorre o seu caminho de forma independente, de acordo com as suas próprias regras e dinâmicas. Em determinados momentos e sob determinadas condições, os fluxos convergem, favorecendo a abertura de uma janela de oportunidade, que "proporciona aos defensores de uma determinada proposta uma possibilidade para fazer valer as suas ideias ou para determinar que a atenção política se centre nos seus problemas específicos" (Kingdon, 2011:165).

Esta convergência é impulsionada principalmente pelo fluxo dos problemas quando um problema consegue captar a atenção política (na sequência do conhecimento e divulgação de indicadores, de resultados de ações políticas, ou da ocorrência de eventos ou crises) - e pelo fluxo da política - quando ocorrem mudanças 
governamentais, pressões públicas ou alterações do sentimento nacional. O fluxo das políticas não tem um efeito tão direto no processo: as alternativas e soluções mantêm-se ativas e atualizadas no seio das comunidades políticas e epistémicas e emergem quando é aberta a janela de oportunidade por influência dos outros dois fluxos, sendo então possível ocorrer mudanças na agenda política.

Essencial para a compreensão do mecanismo de junção dos três fluxos (coupling) é o contexto no qual, segundo Kingdon, decorre o processo político. O papel e o peso dos diferentes atores no decurso do processo, a forma como afetam a disponibilização de alternativas e a escolha de soluções e o modo como utilizam os recursos são decisivos neste modelo.

O modelo Multiple Streams distingue, para efeitos de simplificação da análise, dois grandes conjuntos de atores: (1) o conjunto de atores visíveis (visible cluster), mais exposto à pressão e atenção pública, que inclui o governo, o parlamento e os membros da administração com poder decisório, e (2) o conjunto de atores invisíveis (hidden cluster), composto por grupos de interesse, burocratas, pesquisadores, académicos, partidos políticos, média e opinião pública. Cada um dos conjuntos dispõe de recursos distintos: os atores visíveis dispõem de uma autoridade formal e de prerrogativas legais, que lhes são concedidas pelo seu próprio estatuto, enquanto os atores invisíveis detêm um maior controlo sobre as alternativas e soluções disponíveis. Independentemente de estarem inseridos num ou noutro destes conjuntos, emergem atores (ou grupos de atores) que estão dispostos a investir os recursos de que dispõem - designadamente tempo, energia, reputação, recursos financeiros - para colocar no centro da atenção política os seus problemas e/ou as alternativas que defendem. Kingdon designa estes atores como empreendedores políticos (policy entrepeneurs), que desempenham um papel fundamental no processo, tendo uma grande influência na criação de condições favoráveis à junção dos fluxos, à abertura de janelas de oportunidade e à introdução de mudanças na agenda política.

O modelo Multiple Streams tem sido objeto de propostas de alteração e de introdução de novos fatores e mecanismos de análise, com o propósito de ultrapassar limitações que lhe têm sido apontadas, algumas delas posteriormente reconhecidas pelo próprio Kingdon.

Uma destas críticas prende-se com a conceptualização da autonomia dos fluxos. Alguns autores têm defendido que a análise seria mais proveitosa se fosse considerada a interdependência dos fluxos, em que as mudanças ocorridas num deles tivessem impacto na trajetória dos outros, o que tornaria o processo de coupling mais estratégico, ultrapassando a ideia de imprevisibilidade do processo. ${ }^{14}$ Vários autores consideram que a principal fragilidade do modelo reside na pouca atenção

14 Sabatier e Schlager (2000: 225) consideram que, embora o modelo de Kingdon evidencie pontos fortes, como sejam a atenção dada à envolvente socioeconómica, a centralidade conferida às ideias e à informação, a capacidade de integrar na análise uma diversidade de fatores cognitivos e não cognitivos e a possibilidade de ser replicado em diferentes contextos, este não disponibiliza uma metodologia satisfatória para determinar em que fluxos se integram os atores da ação pública, para além de constituir uma metáfora intuitiva que não cumpre adequadamente critérios de cientificidade. 
que dá ao contexto institucional no qual decorre a ação política, sendo necessário salvaguardar essa análise complementar.

\section{O modelo do equilíbrio interrompido (Punctuated Equilibrium Theory)}

O modelo Punctuated Equilibrium, ${ }^{15}$ desenvolvido por Frank Baumgartner e Bryan Jones, é construído com base no seguinte princípio: os processos políticos são geralmente caracterizados por estabilidade e incrementalismo, pontuado ou interrompido, ocasionalmente, por mudanças de larga escala. A estabilidade, mais que as crises, caracterizam a maioria das áreas de política, mas as crises ocorrem. Compreender o processo político implica analisar as condições de estabilidade e simultaneamente as condições da mudança (True, Jones e Baumgartner, 2007: 155-156).

O objetivo dos autores foi construir uma abordagem que permitisse explicar quer as descontinuidades ou interrupções (punctuations), quer as continuidades (stasis) das políticas, argumentando que o mesmo sistema institucional gera as mudanças pequenas e graduais e as grandes ruturas. Na sua abordagem os autores propõem uma metodologia que combina estudos qualitativos de políticas públicas com estudos quantitativos e longitudinais, seguindo as mudanças de políticas em longos períodos de tempo. Propõem como chave para compreender as mudanças na agenda política, isto é, as interrupções ou descontinuidades dos equilíbrios, dois conceitos: "imagem política" (policy image) e "subsistema político".

Em primeiro lugar, o conceito de imagem política - a forma como uma política é percebida e discutida - e os "processos de mudança nas políticas públicas" dependem da forma como os assuntos são percecionados publicamente. As imagens políticas são ideias que permitem a compreensão dos problemas e das soluções e que podem ser comunicadas de forma simples e partilhadas por uma comunidade, ou seja, são retratos dos problemas, incluindo as narrativas sobre as suas causas e as soluções para os resolver. Quando uma imagem é largamente partilhada e aceite constitui-se como monopólio político. Os monopólios políticos mantêm o equilíbrio e a estabilidade dos sistemas. Quando há divergências em relação a uma imagem os defensores de ideias diferentes podem conseguir desestabilizar o monopólio. Imagens políticas alternativas são desenvolvidas com base em dois tipos de componentes: (1) informações e dados empíricos e (2) apelos emotivos. E são geradoras de disputas para a alteração das políticas.

Os autores analisam com detalhe uma diversidade de problemas para testar o modelo, e concluem que existe um padrão de evolução semelhante: a estabilidade é caracterizada pela prevalência de monopólios políticos, sendo interrompida quando

15 A designação que Baumgartner e Jones dão ao seu modelo analítico alude à teoria da biologia evolutiva com o mesmo nome, que, contrariando a mudança lenta e progressiva das espécies defendida por Darwin, defende a existência de largos períodos de stasis (estabilidade), interrompidos por raros mas intensos períodos de mudança. O suporte empírico do modelo consistiu na classificação e análise de artigos publicados nos meios de comunicação social, e de intervenções nas audiências no Congresso norte-americano e outras informações, sobre as políticas setoriais concretas desenvolvidas ao longo de várias décadas. 
um monopólio é desafiado, destruído, reconstruído ou substituído por outro. Todos os grupos e comunidades têm interesse em estabelecer um monopólio e um arranjo institucional que reforce tal monopólio.

Em segundo lugar, o conceito de subsistema político formado por comunidades de especialistas numa determinada área, nos quais os governos delegam a tarefa de processar as questões políticas. Partindo do princípio da impossibilidade de os governos lidarem em simultâneo com uma grande diversidade de assuntos, o modelo Punctuated Equilibrium defende que é no interior dos subsistemas políticos que são processados os problemas e toda a informação disponível. Os subsistemas políticos são, assim, entendidos como mecanismos que permitem ao sistema político assegurar o "processamento paralelo" (parallel processing) de grandes volumes de informação diversificada. O agendamento traduz a passagem de um problema do subsistema (comunidades de especialistas) para o macrossistema (governo). Tal como Kingdon, Baumgartner e Jones defendem que as questões políticas não se transformam automática e naturalmente em problemas políticos. Para estes autores, é a imagem política que estabelece a ligação entre o problema, a solução e a possibilidade do seu agendamento. A existência de monopólio político exige o exercício de controlo sobre o discurso e a visão dos problemas. Os subsistemas específicos, que adquirem o controlo sobre a interpretação dos problemas e a forma como são discutidos geram feedbacks negativos sobre as agendas, impedindo os processos de mudança. Dentro dos subsistemas, as relações estabelecidas entre grupos com poder para mudar a imagem de determinado problema afetam as dinâmicas de agendamento. Nos subsistemas mais competitivos, a transformação de um monopólio político, com novos atores, com novas ideias, conduz ao agendamento de novos problemas e a novos debates, e depois a novos monopólios e períodos de estabilidade.

O modelo de Baumgartner e Jones constitui-se como uma nova forma de olhar as políticas públicas, na medida em que tem por objetivo não só explicar os longos períodos de estabilidade que caracterizam a ação pública, mas também as rápidas e explosivas mudanças que pontuam o equilíbrio dominante, elegendo os subsistemas políticos como arranjos institucionais que garantem a estabilidade, onde as ideias ocupam um lugar preponderante.

São, no entanto, apontadas algumas limitações ao modelo. John (2013) refere designadamente que: (1) a metodologia utilizada permite identificar a existência de associações, mas não de relações causais entres as agendas dos média, da opinião pública e das arenas políticas com os resultados das políticas; (2) o modelo é essencialmente bottom-up, negligenciando a capacidade que os decisores políticos têm de formatar as decisões de acordo com as suas preferências.

O quadro teórico das coligações de causa ou de interesse (Advocacy Coalition Framework - ACF)

Como vimos, o modelo sequencial tem sido a base a partir da qual se construíram os diferentes modelos de análise das políticas públicas. O modelo Advocacy Coalition Framework (ACF), proposto na década de 1980 por Paul Sabatier e Jenkins-Smith, 
constitui uma das mais importantes formas alternativas de pensar o processo político, propondo-se: (a) encontrar uma alternativa ao modelo heurístico das etapas, que dominava o campo de estudo das políticas; (b) sintetizar os melhores contributos das abordagens top-down e bottom-up, explicativas da implementação de políticas; e (c) incorporar informação técnica nas teorias do processo político. O objetivo foi disponibilizar uma explicação coerente sobre os principais fatores e dinâmicas que afetam o processo político.

Na análise, separar a fase de agendamento das restantes fases do processo político nãoé realista nem permite compreender as mudanças que ocorrem. (Sabatier, 1998: 98)

Em diálogo com os autores da tese do Iron Triangle, amplamente utilizada pela ciência política para explicar a formação de políticas públicas, ${ }^{16}$ Sabatier defende, para compreender o desenvolvimento do processo político, o recurso a vários conceitos: sistema de crenças, subsistema político, coligação de causas e mediadores políticos. O sistema de crenças envolve ideias, valores ontológicos e normas (deep core beliefs), perceções sobre as causas dos problemas e os efeitos e a eficácia das soluções políticas, das instituições e dos recursos mobilizados (policy beliefs), sendo partilhados por atores envolvidos em determinada política.

Os autores consideram que o subsistema político é a unidade de análise mais adequada para analisar o processo político. Este define-se como um conjunto de atores, integrados em organizações públicas e privadas, que se interessam ativamente por uma determinada área de política e tentam influenciar o desenvolvimento político nessa área (Sabatier, 1998: 98). Fazem parte do subsistema todos os que participam e desempenham um papel importante na geração, disseminação e avaliação das ideias políticas em relação a um tema ou área específica: analistas, grupos de interesse, burocratas, políticos eleitos, académicos, think tanks, investigadores, jornalistas e membros de diferentes níveis do sistema político-administrativo e de governo.

Os intervenientes no processo político procuram alianças com atores dos diversos níveis governamentais que partilham ideias políticas semelhantes - legisladores, burocratas, líderes de grupos de interesse, juízes, investigadores e intelectuais (Sabatier, 2007: 196).

É a partilha de um mesmo sistema de crenças que mantém os atores unidos no mesmo subsistema. Quando estes atores agem de forma concertada para atingir determinados objetivos políticos, está formada uma coligação de causa ou de interesse (advocacy coalition). A associação ou a competição entre coligações geram dinâmicas de mudança nas políticas públicas e dinâmicas de recomposição e de emergência de novas questões. Os subsistemas integram uma diversidade de coligações 
que se distinguem umas das outras pelos recursos de que dispõem e pelas ideias políticas (policy beliefs) que defendem (advocacy coalition) e que competem entre si para influenciar a tomada de decisão política.

O modelo prevê a intervenção, no interior dos subsistemas, de mediadores políticos (policy brokers), atores que tentam gerar compromissos entre as posições das diferentes coligações, com o objetivo de apresentar propostas de mudança política viáveis e influenciar a posição dos decisores políticos e das instituições governamentais. Esta capacidade de influenciar e promover a mudança reflete-se nos resultados das políticas (policy outputs), que por sua vez têm impacto, através de processos de feedback, nas ideias políticas e nos recursos das coligações, processo que os autores designam policy oriented learning e definem como alterações relativamente duradouras de pensamento ou de comportamento, que resultam da experiência de ações políticas anteriores e/ou da aquisição de nova informação (Sabatier, 2007: 198).

Os autores defendem que dois tipos de variáveis têm impacto ao nível do equilíbrio de poder e da distribuição de recursos no interior do subsistema: variáveis estáveis (estrutura social, regras, instituições, valores e recursos do sistema político) e variáveis instáveis ou eventos exteriores (mudanças socioeconómicas, mudanças no sistema de governo, mudanças ou decisões em outros subsistemas). Mas, ao contrário de outras abordagens que valorizam a influência do contexto e das pressões externas no desenvolvimento do processo político (como é o caso do modelo de Kingdon, que dá relevo ao papel de fatores externos ao processo político, como o sentimento nacional, o turnover governamental e a pressão dos grupos de interesse), os autores do ACF defendem que "eventos externos ao subsistema são condição necessária, mas não suficiente, para a mudança nas políticas" (Sabatier, 2007: 199), valorizando antes a importância das ideias políticas, quer na formação e desenvolvimento das coligações, quer como fator indutor de mudança política.

Os autores preveem ainda a existência de uma terceira variável, exercendo funções de mediação entre as outras duas e o subsistema, condicionando e enquadrando o comportamento dos atores e das suas interações: esta variável, designada long term coalition opportunity structure, diz respeito ao "grau de consenso necessário à mudança política" e ao "grau de abertura do sistema político". Defendem os autores que uma estrutura que promova e incentive altos graus de consenso e de partilha de informação entre as diferentes coligações e privilegie um fácil acesso aos patamares decisórios contribui para minimizar os riscos de conflito e para alcançar os objetivos políticos.

O modelo proposto por Sabatier e Jenkins foi elaborado com o propósito mais abrangente de encontrar uma explicação para o processo político alternativa ao modelo das etapas. Nesse sentido, para além de proporcionar uma abordagem mais integrada dos processos de emergência e de formulação das políticas públicas, propõe também uma síntese das abordagens top-down e bottom-up da implementação de políticas. A abordagem top-down defende que um processo eficaz de implementação requer uma "cadeia de comando" com capacidade para coordenar e controlar o processo, enquanto que a abordagem bottom-up valoriza a influência das rotinas e dos procedimentos das administrações (street level bureaucracy) e dos 
expedientes utilizados para lidar com situações de incerteza como um fator determinante para o sucesso ou insucesso da concretização de políticas.

O quadro analítico Advocacy Coalition Framework tem sido utilizado, com sucesso para a análise de processos de implementação. ${ }^{17} \mathrm{Na}$ sequência da experiência resultante da aplicação do modelo em diversas pesquisas empíricas e do esforço para sintetizar as teorias até então dominantes, Sabatier (em conjunto com David Mazmanian) apresentou um conjunto de seis condições necessárias e suficientes para o desenvolvimento adequado e eficaz do processo de implementação (em Parsons, 1995: 486): (i) objetivos claros e consistentes, que permitam estabelecer um padrão de avaliação normativa e de recursos; (ii) uma adequada teoria causal, que assegure que a política incorpora uma abordagem para induzir a mudança; (iii) uma estrutura legalmente enquadrada, que favoreça o compromisso entre os responsáveis pela implementação e os grupos-alvo da política; (iv) responsáveis pela implementação competentes tecnicamente e empenhados em atingir os objetivos da política; (v) apoio de grupos de interesse e de titulares de altos cargos no parlamento e no governo; (vi) mudanças nas condições socioeconómicas, por forma a não comprometer o apoio dos grupos de interesse, parlamentares e governantes e a não subverter a teoria causal subjacente à política.

Ainda de acordo com Parsons (1995: 487), este modelo, embora mais associado à abordagem top-down, ao defender que as coligações que integram atores dotados de autoridade formal são, regra geral, as coligações dominantes dentro do subsistema político - o que é, aliás reconhecido pelo próprio Sabatier (2007: 201-202) - representa uma efetiva sintese das duas abordagens referidas, uma vez que enfatiza a importância do papel das elites políticas e da existência de uma linha hierárquica de coordenação, mas simultaneamente incorpora preocupações da abordagem bottom-up, ao valorizar também o papel das burocracias e das estruturas de implementação.

\section{Notas conclusivas}

Neste texto passámos em revista os contributos teóricos dos fundadores da análise das políticas públicas e apresentámos quatro modelos teóricos de análise: (1) o modelo sequencial ou do ciclo político, (2) o modelo dos fluxos múltiplos, (3) o modelo do equilíbrio interrompido e (4) o quadro teórico das coligações de causa ou de interesse. Como referimos, são quadros explicativos do funcionamento das políticas públicas considerados por vários autores como sendo, dadas as suas características, quadros analíticos promissores. Trata-se de modelos teóricos logicamente coerentes, baseados em proposições empiricamente verificáveis, claros e abrangentes, replicáveis em áreas de política, situações e contextos distintos. Apresentam-se como modelos de síntese, uma vez que promovem a integração de conceitos e propostas analíticas de outros autores, explorando a complementaridade das diferentes abordagens.

17 Segundo Parsons (1995: 486), o modelo foi utilizado para analisar uma grande diversidade de casos nos EUA e na Europa, revelando-se de grande utilidade para orientar a pesquisa empírica da implementação de políticas. 
Procurámos assim contribuir para melhorar as condições de desenvolvimento deste campo disciplinar também em Portugal. ${ }^{18}$ No atual contexto, a análise das políticas públicas tem uma importância renovada, porque pode ser um instrumento de promoção da qualidade das políticas públicas, da exigência, do rigor e da transparência dos gastos públicos.

Porém a análise das políticas públicas pode permitir também compreender melhor algumas das tensões das tendências contraditórias que atravessam os debates públicos na sociedade portuguesa. Quer seja o debate público sobre o papel do estado, a sua dimensão e a natureza da sua intervenção, revelando tensões entre, por um lado, os defensores de políticas keynesianas e de um papel ativo do estado, não apenas na regulação mas também na resolução de problemas que o mercado não resolve, com investimento público e respondendo a crescentes necessidades e expectativas dos cidadãos; e, por outro lado, os defensores de políticas restritivas e de um estado mínimo, não interventor, privilegiando a ação dos mercados e a iniciativa individual, pressionando para a redução do défice, da dívida e do investimento público. Quer seja o debate sobre as questões da soberania e o efetivo poder dos governos nacionais, partindo do reconhecimento das dinâmicas da globalização e da transnacionalização, da influência de fatores externos e da intervenção de organismos internacionais e transnacionais, como a OCDE, o FMI e as instituições da UE, tem suscitado interrogações sobre o funcionamento da democracia nos estados-membros.

18 Com objetivo similar, vários trabalhos têm vindo a ser publicados sobre as políticas públicas em Portugal, por M. L. Rodrigues e P. Adão e Silva (2013; 2014; 2015). 


\section{Anexo}

Quadros teóricos, abordagens e modelos de análise de políticas públicas

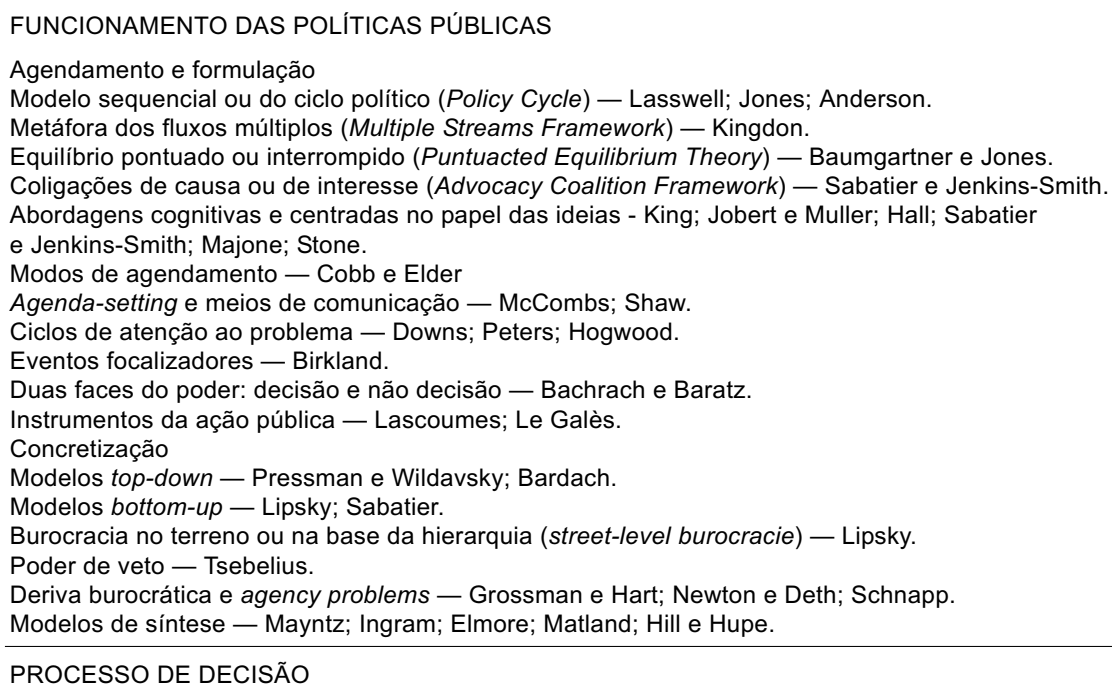




\section{Referências bibliográficas}

Anderson, J. E. (2003), Public Policymaking, Boston, Houghton Mifflin.

Baumgartner, Frank R., Martial Foucault, e Abel François (2012 ), “Public budgeting in the EU Commission: a test of the Punctuated Equilibrium Thesis", Politique Europpéenne, 38, pp. 70-99, disponível em: http://www.unc.edu/ fbaum/articles/ PE-in-EU-Budgets-2012.pdf (última consulta em agosto de 2016).

Birkland, Thomas A. (1978) "Focusing events, mobilization, and agenda setting". Jourmal of Public Policy, 18 (1), pp. 53-74.

Birkland, Thomas A. (2011), An Introdution to the Policy Process, Theories, Concepts and Models of Public Policy Making, Nova Iorque e Londres, M. E. Sharpe (3. a edição).

Capella, Ana Cláudia N. (2006), “Perspectivas teóricas sobre o processo de formulação de políticas públicas", Revista Brasileira de Informação Bibliográfica em Ciências Sociais, 62, pp. 25-52, disponível em: http://www.anpocs.org.br/portal/images/bib61.pdf (última consulta em agosto de 2016).

Cohen, Michael D., James G. March, e Johan P. Olsen (1972), “A Garbage Can Model of organizational choice", Administrative Science Quarterly, 17 (1), pp. 1-25, disponível em: http://links.jstor.org/sici?sici=0001-8392\%28197203\%2917\%3A1\%3C1\%3AAGCMOO\% 3E2.0.CO\%3B2-9 (última consulta em agosto de 2016).

DeLeon, Peter (2006), "The historical roots of the field", em Michael Moran et al. (orgs.), The Oxford Handbook of Public Policy, Oxford, Oxford University Press. pp. 39-57.

DeLeon, Peter, e Danielle M. Vogenbeck (2007), "The policy sciences at the crossroads", em Frank Fischer, Gerald J. Miller e Mara S. Sidney (orgs.), Handbook of Public Policy Analysis. Theory, Politics and Methods, Boca Raton, FL, CRC Press, pp. 3-14.

Dobbin, Frank (1994), Forging Industrial Policy. The United States, Britain, and France in the Railway Age, Cambridge (etc.), Cambridge University Press.

Dye, Thomas R. (2001), Top Down Policymaking, Nova Iorque, Chatham House, Even Bridges Press.

Dye, Thomas R. (2010), Understanding Public Policy, Boston (etc.), Longman (13. e edição).

Easton, David (1957), "An aproach to the analysis of political systems", World Politics, 9 (3), pp. 383-400, disponível em: http://web.unair.ac.id/admin/file/f_23123_ System-Easton.pdf (última consulta em agosto de 2016).

Fisher, Frank (2003), Reframing Public Policy. Discursive Politics and Deliberative Pratices, Oxford e Nova Iorque, Oxford University Press.

Haas, Peter. (1992), “Introduction: epistemic communities and international policy coordination", International Organization, 46 (1) (número temático: Knowledge, Power and International Coordination), pp. 1-35, disponível em: http://links.jstor.org/sici?sici=0020-8183\%28199224\%2946\%3A1\%3C1\%3AIECAIP\% 3E2.0.CO\%3B2-\%23 (última consulta em agosto de 2016).

Hall, Peter (1993), "Policy paradigm, social learning and the state: the case of economic policymaking un Britain", Comparative Politics, 25 (3), pp. 275-296, disponível em: http://chenry.webhost.utexas.edu/core/Course\%20Materials/Hall/0.pdf (consultado em 24/02/2015).

Hall, Peter, e Rosemary C. R. Taylor (1996), “Political science and the three new institutionalisms", Political Studies, 44, pp. 936-957, disponível em: 
http://chenry.webhost.utexas.edu/core/Course\%20Materials/Hall\%26TaylorPolStu ds/9705162186.pdf (consultado em 26/03/2013).

Heidenheimer, Arnold J., Hugh Heclo, e Carolyn Teich Adams (1975), Comparative Public Policy. The Politics of Social Choice in Europe and America, Nova Iorque, St. Martin's Press, disponível em: http://www.questia.com/read/91228549/comparative-publicpolicy-the-politics-of-social (última consulta em agosto de 2016).

Hill, Michael (2009), The Public Policy Process, Harlow, Pearson Education (5. edição).

Hill, Michael, e Peter Hupe (2009), Implementing Public Policy. An Introduction to the Study of Operational Governance, Londres (etc.), Sage Publications (2. $\cdot^{\mathrm{a}}$ edição).

Hogwood, B., e L. Gunn (1984), Policy Analysis for the Real World, Oxford, Oxford University Press.

Howlett, M., M. Ramesh, e A. Perl (2009), Studying Public Policy. Policy Cycles E Policy Subsystems, Oxford, Oxford University Press.

Immergut, Ellen M. (1992), Health Politics. Interests and Institutions in Western Europe, Cambridge, UK, Nova Iorque e Melbourne, Cambridge University Press.

Immergut, Ellen M. (2006), "Institutional constraints on policy", em Michael Moran et al., The Oxford Handbook of Public Policy, Oxford, Oxford University Press, pp. 557-571.

Jenkins, W. (1978), Policy Analysis. A Political and Organizational Perspective, Oxford, Blackwell.

John, Peter (1998), Analysing Public Policy, Londres, Continuum.

John, Peter (2013), "New directions in public policy: theories of policy change and variation reconsidered", comunicação apresentada na International Conference on Public Policy, Grenoble, junho de 2013, disponível em: http://www.icpublicpolicy.org/IMG/pdf/panel_84_1_peter_john.pdf (última consulta em agosto de 2016).

Jones, C. O. (1984), An Introdution to the Study of Public Policy, Monterey, CA, Brooks Cole Publishing (3. ․ edição).

Kingdon, John W. (2011), Agendas, Alternatives and Public Policies, Boston, Longman (2.. edição revista).

Knoepfel, Peter, et al. (2011), Public Policy Analysis, Bristol, UK, The Policy Press.

Kraft, Michael E., e Scott R. Furlong (2010), Public Policy, Politics, Analysis and Alternatives, Washington, DC, CQ Press (3. a edição).

Lasswell, Harold D. (1948), The Analysis of Political Behavior. An Empirical Approach, Londres, Routledge and Kegan Paul.

Lasswell, Harold D. (1956), The Decision Process. Seven Categories of Functional Analysis, College Park, MD, University of Maryland Press.

Lasswell, Harold D. (1970), "The emerging conception of the policy sciences", Public Policy, 1, pp. 3-14.

Levi, M. (2009), “A model, a method and map: racional choice in comparative and historical analysis", em M. Lichbach e A. Zuckerman (orgs.), Comparative Politics. Racionality, Culture and Structure, Cambridge, Cambridge University Press. pp. 1-17 (2.. edição).

Lindblom, Charles E. (1959), "The science of 'muddling through' ", Public Administration Review, 19 (2), pp. 79-88, disponível em:

https://faculty.washington.edu/mccurdy/SciencePolicy/Lindblom $\% 20$ Muddling\%2 0Through.pdf (última consulta em agosto de 2016). 
Lindblom, C., e E. Woodhouse (1993), The Policy-Making Process, Englewood Cliffs, NJ, Prentice Hall.

March, James G., e Johan P. Olsen (1984), “The new institucionalism: organizational factors in political life", The American Political Science Review, 78 (3), pp. 734-749, disponível em: http://online.sfsu.edu/sguo/Renmin/June\%2021_institutionalism/

New\%20Institutionalism_James\%20March.pdf (última consulta em agosto de 2016).

March, James G., e Johan P. Olsen (1996), “Institutional perspectives on political institutions", Governance: An International Journal of Policy and Administration, 9 (3), pp. 47-64, disponível em: http://onlinelibrary.wiley.com/doi/10.1111/ j.1468-0491.1996.tb00242.x/abstract (última consulta em agosto de 2016).

May, J. V., e A. Wildavksy (orgs.) (1978), The Policy Cycle, Beverly Hills, CA, Sage Publications.

Muller, Pierre (2005), “Esquisse d'une théorie de changement dans l'action publique: structures, acteurs et cadres cognitifs", Revue Française de Science Politique, 55, Presses de Sciences Po, pp. 155-187, disponível em: http://www.gare.cree-inter.net/sites/default/files/Esquisse d'une théorie du changement dans l'action publique.pdf (última consulta em agosto de 2016).

Muller, Pierre (2010), Les Politiques Publiques, Paris, Presses Universitaires de France (8. a edição).

Neiman, Max, e Stephen J. Stambough (1998), “Rational choice theory and the evaluation of public policy", Policy Studies Journal, 26 (3), pp. 449-465, disponível em http://www.questia.com/library/1G1-54205080/rational-choice-theory-and-the-eval uation-of-public (consultado em 02/04/2013).

Palier, Bruno, e Yves Surel (2005), “Les 'trois I' et l'analyse de l'État en action”, Revue Française de Science Politique, 55, pp. 7-32, disponível em: www.cairn.info/revue-francaise-de-science-politique-2005-1-page-7.htm (última consulta em agosto de 2016).

Parsons, Wine (1995), Public Policy. An Introduction to the Theory and Pratice of Policy Analysis, Cheltenham, UK, e Northampton, MA, Edward Elgar.

Pierson, Paul (2000), "Increasing returns, path dependence, and the study of politics", The American Political Science Review, 94 (2), pp. 251-267, disponível em: http://www.la.utexas.edu/users/chenry/core/Course\%20Materials/PiersonWk4APS R2000/0.pdf (última consulta em agosto de 2016).

Pierson, Paul (2004), Politics in Time, History, Institutions and Social Analysis, Princeton e Oxford, Princeton University Press.

Pierson, Paul, e Theda Skocpol (2002), "Historical institutionalism in contemporary political science", em I. Katznelson e H. V. Milner, Political Science. State of the Discipline, Nova Iorque, W. W. Norton, pp. 693-721, disponível em: https://www.academia.edu/4711753/HISTORICAL_INSTITUTIONALISM_IN_CO NTEMPORARY_POLITICAL_SCIENCE (última consulta em agosto de 2016).

Rodrigues, Maria de Lurdes (coord.) (2014), Exercícios de Análise de Políticas Públicas, Lisboa, Imprensa Nacional Casa da Moeda.

Rodrigues, Maria de Lurdes, e Pedro Adão e Silva (orgs.) (2013), Políticas Públicas em Portugal, Lisboa, Imprensa Nacional Casa da Moeda.

Rodrigues, Maria de Lurdes, e Pedro Adão e Silva (orgs.) (2014), Políticas Públicas para a Reforma do Estado, Coimbra, Edições Almedina. 
Rodrigues, Maria de Lurdes, e Pedro Adão e Silva (orgs.) (2015), Governar com a Troika. Políticas Públicas em Tempos de Austeridade, Coimbra, Edições Almedina.

Sabatier, Paul A. (1998), “The Advocacy Coalition Framework: revisions and relevance for Europe", Journal of European Policy, 51, pp. 98-130, disponível em: http://pubget.com/paper/pgtmp_1819b4e572a4dd1aaf9ab3dc9175ee63/The_advocacy_co alition_framework_revisions_and_relevance_for_Europe (consultado em 06/10/2011).

Sabatier, Paul A. (org.) (2007), Theories of the Policy Process, Boulder, CO, Westview Press. Sabatier, Paul, e Edella Schlager (2000), “Les approches cognitives des politiques publiques: perspectives américaines", Revue Française de Science Politique, 50 (2), pp. 209-234, disponível em: http://www.persee.fr/doc/rfsp_0035-2950_2000_ num_50_2_395465 (última consulta em 12/07/2016).

Scott, John (2001), Power, Cambridge, UK, Polity Press.

Simon, Herbert (1959), "Theories of decision-making in economics and behavioral science", American Economics Review, 49, pp. 245-283.

Simon, Herbert (1983), "On the behavioural and rational foundation of economic theory", Working Papers Series, 115, Research Institute of Industrial Economics.

Souza, Celina (2006), "Políticas Públicas: uma revisão da literatura" Sociologias, 16, pp. 20-45, disponível em: http://www.scielo.br/pdf/soc/n16/a03n16 (última consulta em 12/07/2016).

Surel, Yves (1998), "Idées, interêts, institutions dans l'analyse des politiques publiques", Pouvoirs, 87, pp. 161-178, disponível em http://www.revue-pouvoirs.fr/IMG/pdf/87Pouvoirs_p161-178_chroniques_analyse _politiques_publiques.pdf (última consulta em 12/07/2016).

Thelen, Kathleen (2004), How Institutions Evolve. The Political Economy of Skills in Germany, Britain, The United States and Japan, Cambridge (etc.), Cambridge University Press.

Torgerson, D. (2007), "Promoting the policy orientation", em Frank Fischer, Gerald J. Miller e Mara S. Sidney (orgs.), Handbook of Public Policy Analysis. Theory, Politics and Methods, Boca Raton, FL, CRC Press, pp. 15-28.

True, James L., Bryan D. Jones, e Frank R. Baumgartner (2007), “Punctuated Equilibrium Theory: explaining stability and change in public policymaking", em Paul A. Sabatier (org.) (2007), Theories of the Policy Process, Boulder, CO, Westview Press, pp. 155-187.

Tsebelis, George (1995), “Decision making in political systems: veto players in presidentialism, parliamentarism, multicameralism and multipartyism", British Journal of Political Science, 25 (3), pp. 289-325.

Tsebelis, George (2000), "Veto players and institutional analysis", Governance: An Intitutional Journal of Policy and Administration, 13 (4), Malden e Oxford, Blakwell Publishing, pp. 441-474.

Tsebelis, George (2001), Veto Players. How Political Institutions Work, Princeton, Princeton University Press e Russell Sage Foundation, disponível em: http://politics.as.nyu.edu/docs/IO/4756/tsebelis_book.pdf (última consulta em 12/07/2016).

Zahariadis, Nikolaos (2007), “The multiple streams framework: structures, limitations, prospects", em Paul A. Sabatier (org.), Theories of the Policy Process, Boulder, CO, Westview Press, pp. 65-92. 
Luísa Araújo. Vogal da direção do FPP - Fórum das Políticas Públicas, Rua

Duque de Palmela, n. ${ }^{\circ}$ 27, 5..$^{\circ}$ esquerdo, Lisboa, Portugal.

E-mail: mlluisa.araujo@gmail.com

Maria de Lurdes Rodrigues (corresponding author). Docente no Instituto

Universitário de Lisboa (ISCTE-IUL) e investigadora no Centro de Investigação e Estudos de Sociologia (CIES-IUL), Lisboa, Portugal.

E-mail: mlreisrodrigues@gmail.com

Receção: 19 de maio de 2016 Aprovação: 1 de agosto de 2016 
